\title{
GENERALISED CAPACITY CURVES FOR STABILITY AND PLASTICITY: APPLICATION AND LIMITATIONS
}

\author{
by Cornelia Doerich and J. Michael Rotter \\ University of Edinburgh, Edinburgh, Scotland, UK
}

\section{Abstract}

In recent decades, the resistance of a structure has been thought of as well defined by the outcome of a geometrically and materially nonlinear analysis with explicitly modelled imperfections (GMNIA). But when this is the only analysis that is performed on a complex structural system, it is sometimes difficult to interpret the result. The outcome must be seen in the context of those from simpler analyses, which can define appropriate reference quantities.

Other analyses, like a small displacement theory materially nonlinear analysis (MNA) and a linear elastic bifurcation analysis (LBA) are very important in the interpretation of a GMNIA.

The general capacity curve in the Eurocode for shell structures [1] provides a representation of these different analyses. Using this capacity curve, different identifiable key aspects of the structure's behaviour can be studied independently and understood in relation to the corresponding parameter of this curve. This unified representation allows an easy and meaningful characterisation of all elastic-plastic buckling problems. 
However, some care is needed when applying such a generalised curve to structures with particular features. This paper outlines the limitations of the simplest version of the curve, and develops an enhancement that permits it to be deployed without restriction.

Keywords: Shell structures, capacity curve, Eurocode, restrictions, nonlinear buckling, plastic collapse

\section{INTRODUCTION}

The capacity curve, presenting the interaction between plasticity and stability in a structural mechanics problem, is a powerful tool for both design and research. It describes the practical compressive resistance of a structural system or component in terms of its slenderness. Such curves, often referred to as a "column curve" though applied to other structures, are normally empirically devised for each structure and load case separately. However, the recent development of a generalised capacity curve [1] that can be used for a wide variety of different structures has opened new opportunities. This curve is expressed in terms of a small number of key parameters, each of which relates to a specific aspect of the structural behaviour, so these parameters can be used to gain deeper insights into the controlling phenomena for the structure [2].

This paper describes the background to the generalised capacity curve adopted into EN 1993-1-6 [1], and shows its usefulness in application to several shell structures. However, some care is needed when applying such a generalised curve to structures with particular and perhaps unusual features, such as yielding at a very low proportion of the plastic failure resistance or structural systems in which yielding and buckling occur in very different locations. This paper outlines the limitations of the simplest version of the curve, and develops an enhancement that permits it to be deployed without restriction.

It is hoped that this generalised capacity curve will be widely adopted for studies of all 
structural systems and forms, so that the strong reliance on special case empiricism that currently dominates this part of structural design can be reduced, and new insights can be gained into the structural behaviour of complex systems.

The capacity curve describes the behaviour of a structural system in terms of two key resistances, as defined by EN 1993-1-6 [1]. The first of these is the lowest elastic linear eigenvalue, derived from a linear elastic small displacement theory bifurcation analysis (LBA). The second is a small displacement theory plastic limit analysis using ideal elasticplastic material properties, which identifies the plastic collapse load of the system (MNA). These two loads are the only two limiting loads that can always be determined for any structural system, since all other analyses may shown indefinitely hardening behaviour and be difficult to interpret [3].

For complex systems it is very difficult to be certain of calculating the true maximum load using a fully geometrically and materially nonlinear analysis with explicit imperfections (GMNIA) since the identification of appropriate imperfection forms and amplitudes can lead to a very tedious search. So it cannot be expected that GMNIA will be used in design, and the drafting panel for EN 1993-1-6 [1] devised instead a methodology termed MNA-LBA in which only the two resistances defined above would be calculated numerically and the remaining phenomena deduced from the expected form of the capacity curve. This method places special importance on the parameters of the capacity curve. In structural framework design, this method has been adopted, with modifications, into [4] and termed the "General Method", but it too relies on the parameters of the capacity curve being known a priori. These developments make the general capacity curve described in this paper of even more importance since some of the parameters within it could potentially be deduced from simpler analyses than a full GMNIA. A fuller description of this methodology is given in [5]. 


\section{The traditional capacity curve}

The concept behind the capacity curve was probably first proposed by Rankine [6] when he identified the two limits of column strength as governed by material failure and by elastic buckling, with his interaction between them providing the first such curve. Later researchers [7-12] expanded on the concept, developing different theoretical backgrounds that continue to be used up to the present day. Comparable curves have been used for beams, plates and shells in the many years since these ideas were first proposed, but only limited and rather crude attempts have been to apply them to structural systems [13],until the development described here.

One unfortunate historical feature of the column curve that is the original basis of this interaction between plasticity and stability is the struggle that occurred between the proponents of different theoretical bases in the 1950s. The followers of Ayrton and Perry [7] adopted the concept of an imperfect column, but had to use first yield as a criterion of failure. By contrast the followers of Considère [8] and Engesser [9] believed in a perfect column and used tangent modulus theory to determine when bifurcation would occur. The critics of the former pointed out that structures yield a lot before failure so it had a poor basis in mechanics, whilst the critics of the latter pointed out that all structure were imperfect so this inelastic theory idealised the structure excessively. The adoption of a Perry treatment in the EN 19931-1 [4] unfortunately merely adopts one of these positions and does not move the debate onwards. The curve of this paper attempts to avoid such sterile arguments.

The essential feature of these capacity curves is that two limiting cases are clearly defined: one based on pure material failure, the other based on elastic stability failure, and an interposing relation between the two is defined, using the "slenderness" of the structure as the measure between them. The general expression for slenderness $\lambda$ is derived from columns as: 
$\lambda=\sqrt{\frac{R_{p l}}{R_{c r}}}$

where $R_{p l}$ is the resistance to material failure (plasticity) and $R_{c r}$ is the elastic critical resistance. For a structural system subject to a complex load set, it is not possible to define these resistances in terms of a force or bending moment, torque or bimoment or stress, since none of these can capture the complete state of the structure. Instead, the design load condition of the structure must be defined, and analyses can be performed to see how much those loads can be increased before failure in a particular mode. The resulting load incrementation factors are the structure's resistance in that mode to that load set, and are consequently defined as $R$ in Rotter and Schmidt [5] .

\section{The new generalised capacity curve}

The recent development of a generalised capacity curve [1] that can be used for a wide variety of different structures and structural systems has opened new opportunities. As noted in the introduction, this curve is expressed in terms of a small number of key parameters, each of which relates to a specific aspect of the structural behaviour, so these parameters can be used to gain deeper insights into the controlling phenomena for the structure [2]. These key parameters represent the major advantage of the generalised capacity curve over the earlier empirical column curves.

This capacity curve, proposed by [14], describes the complete behaviour of a structure from a fully plastic collapse at a low slenderness, $\lambda=\lambda_{0}$, through plastic buckling, $\lambda_{0}<\lambda<\lambda_{\mathrm{p}}$, to elastic buckling including imperfections at high slenderness $\left(\lambda>\lambda_{\mathrm{p}}\right)$ using the relative slenderness $\lambda$ of the structure (Figure 1) and very few parameters to define the failure behaviour of the structure. 


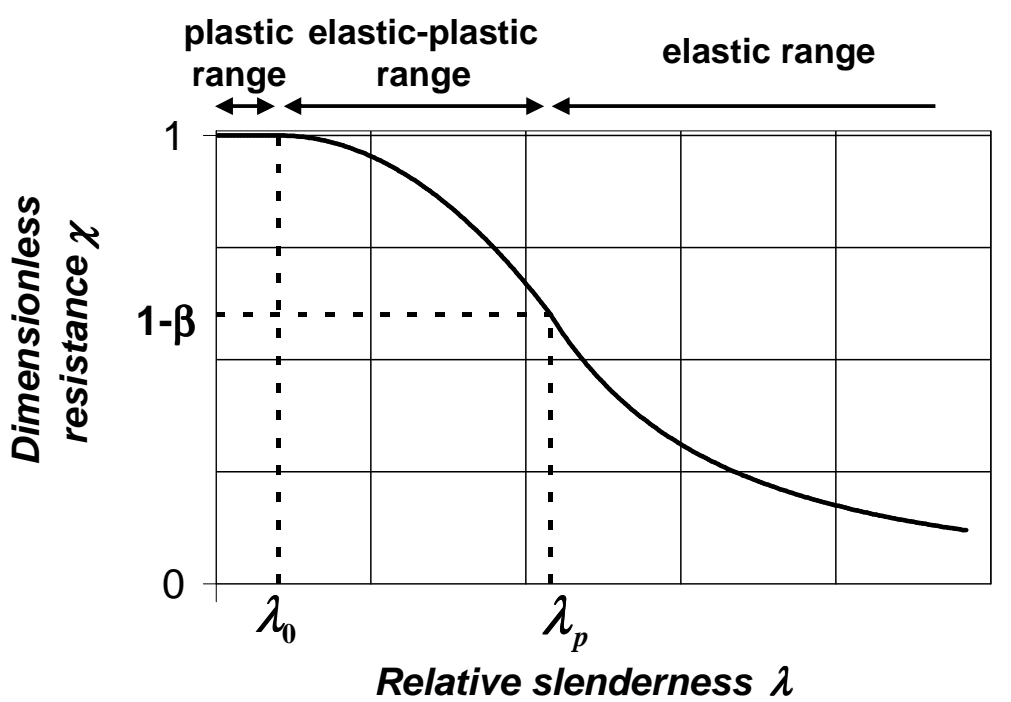

Figure 1: Generalised capacity curve

The relative slenderness of the structural system $\lambda$ is still defined by Eq.1, with $R_{p l}$ as the plastic limit resistance derived from $\left(R_{M N A}\right)$ from a small displacement theory ideal elasticplastic analysis (MNA) and $R_{c r}$ is the elastic critical resistance $\left(R_{L B A}\right)$ from a linear bifurcation analysis (LBA).

The dimensionless resistance parameter $\chi$ is defined as

$\chi=R_{f} / R_{p l}$

where the dimensionless resistance $R_{f}$ is the failure load factor, found in an experiment or calculated using a geometrically and materially nonlinear analysis of the imperfect structure $\left(R_{G M N I A}\right)$

The shape of the capacity curve of EN 1993-1-6 [1] is given by

$\chi=1$

$\chi=1-\beta\left(\frac{\bar{\lambda}-\bar{\lambda}_{0}}{\bar{\lambda}_{p}-\bar{\lambda}_{0}}\right)^{\eta}$

$\chi=\alpha / \bar{\lambda}^{2}$ when

when

when 
with

$$
\bar{\lambda}_{p}=\sqrt{\alpha /(1-\beta)}
$$

Equations (3-6) were originally proposed by [14]. Equation 3 was later modified [3] to include a hardening zone as

$\chi=\chi_{h}-\left(\bar{\lambda} / \bar{\lambda}_{0}\right)\left[\chi_{h}-1\right] \quad$ when $\quad \bar{\lambda} \leq \bar{\lambda}_{0}$

The capacity curve has three sections: in the first (Eqs 3 or 7) the resistance is equal to or exceeds the plastic limit resistance $R_{p l}$ (derived from an MNA analysis) for slendernesses smaller than the squash limit relative slenderness $\lambda_{0}$.

Resistance above $R_{p l}$ are found in all numerical analyses if any one of the following three features is present: a) strain hardening is included, b) changes in geometry (geometric hardening) raises the load above the plastic collapse load $R_{p l}$, c) a 3D stress state (e.g. at restrained ends) leads to an increase in the applied stress (e.g. the maximum of Mises envelope at $\left.1.15 \sigma_{\mathrm{y}}\right)$. Calculations that do not include any one of these features cannot exceed $R_{p l}$, so no plateau can exist. The existence of this plateau in EN 1993-1-1 [4] derives from experimental results that display resistances significantly greater than $R_{p l}$. Furthermore, metals without a marked yield plateau (aluminium or stainless steel) must be characterised by the $0.2 \%$ proof stress, so structures composed of them always display strain hardening and exceed the plastic limit load based on the proof stress.

In the moderate slenderness part of the capacity curve (Eq. 4), which extends from the squash limit relative slenderness $\lambda_{0}$ to the plastic limit relative slenderness $\lambda_{p}$, plasticity and stability phenomena interact. In the slender section, with slendernesses greater than the plastic limit relative slenderness $\lambda_{p}$, failure occurs by elastic stability alone, but may be strongly influenced by imperfections that reduce the elastic buckling resistance (Eq. 5). 
Only four parameters are needed to describe the complete capacity curve: the squash limit relative slenderness $\lambda_{0}$, the plastic range factor $\beta$, the elastic imperfection factor $\alpha$ and the interaction exponent $\eta$. It should be noted that three of these parameters are not simply empirical fits, but each describes a distinct feature of the structural behaviour of the system.

The limiting slenderness for the plastic plateau is the squash limit relative slenderness $\lambda_{0}$, which defines how stocky a structure must be to attain the fully plastic limit resistance $R_{p l}$. This slenderness $\lambda_{0}$ thus indicates the point at which stability effects first cause reductions in resistance.

At the other extreme, the elastic buckling behaviour of the structure is governed by the elastic imperfection reduction factor $\alpha$, which captures both the effects of imperfections and of changes of geometry (geometric nonlinearity). By re-arranging Eq. 5, $\alpha$ can be expressed as

$\alpha=\frac{R_{G N I A}}{R_{c r}}$

where $R_{\text {GNIA }}$ is the load factor found in an elastic geometrically nonlinear analysis with explicit account of imperfections and the elastic critical resistance $R_{c r}$ is still the result of a linear bifurcation analysis $\left(R_{L B A}\right)$.

The terminology for $\alpha$ (elastic imperfection reduction factor) is convenient for structures like the widely studied case of an axially compressed cylinder where imperfections lead to a drastic decrease in resistance, and geometric nonlinearity plays only a minor role. In such cases, the elastic imperfection factor $\alpha$ is close to 1 for the perfect structure, but decreases rapidly when imperfections are introduced.

The same is not true for many other shell structures, where geometric nonlinearity may yield an "elastic imperfection factor" as low as 0.5 for the perfect structure (e.g. [15]), but the 
subsequent introduction of imperfections may not affect the resistance significantly. For these structures, the elastic critical resistance $R_{L B A}$ is not achieved in the perfect elastic structure and an elastic geometrically nonlinear analysis produces a load factor $R_{G N A}$ that falls far short of the elastic critical resistance $R_{L B A}$. A classic example of this is elastic snapthough buckling in arches and the lids of food containers. For these structures, $\alpha$ must serve the role of indicating the effect of changes of geometry too.

The decision to name the parameter $\alpha$ as the elastic imperfection factor $\alpha$ may therefore be misleading. This parameter must be evaluated carefully in order to distinguish between the two phenomena (geometric nonlinearity and imperfection sensitivity) since the geometric imperfections can be significantly reduced by good fabrication practice, but the geometrically nonlinear effect cannot be avoided.

Where material nonlinearity is also included in this analysis (GMNIA), a very similar load factor $R_{G M N I A} \approx R_{\text {GNIA }}$ is sometimes achieved. This situation indicates that either no yielding has occurred, or that the yielding is in a location where it does not affect the buckling condition, or that yielding in the buckling zone has a small influence on the buckling resistance. There is an important distinction here, because the calculated elastic buckling load is not limited by the condition of first yield, but by the point at which yielding in the structure first sufficiently affects the resistance to be required to be included in the design process. These two points are often quite different.

The resistance $\chi$ at the slenderness where plasticity first causes an identifiable reduction in the elastic buckling resistance is denoted in the capacity curve by $(1-\beta)$, where $\beta$ is the plastic range factor.

In the slenderness range where plastic buckling occurs, the capacity curve can take a variety of different shapes, depending on whether plasticity has a strong effect or only a minor 
effect, as illustrated in Figure 2. To capture these differences, the interaction exponent $\eta$ describes the rate of change of resistance with the slenderness of the structure.

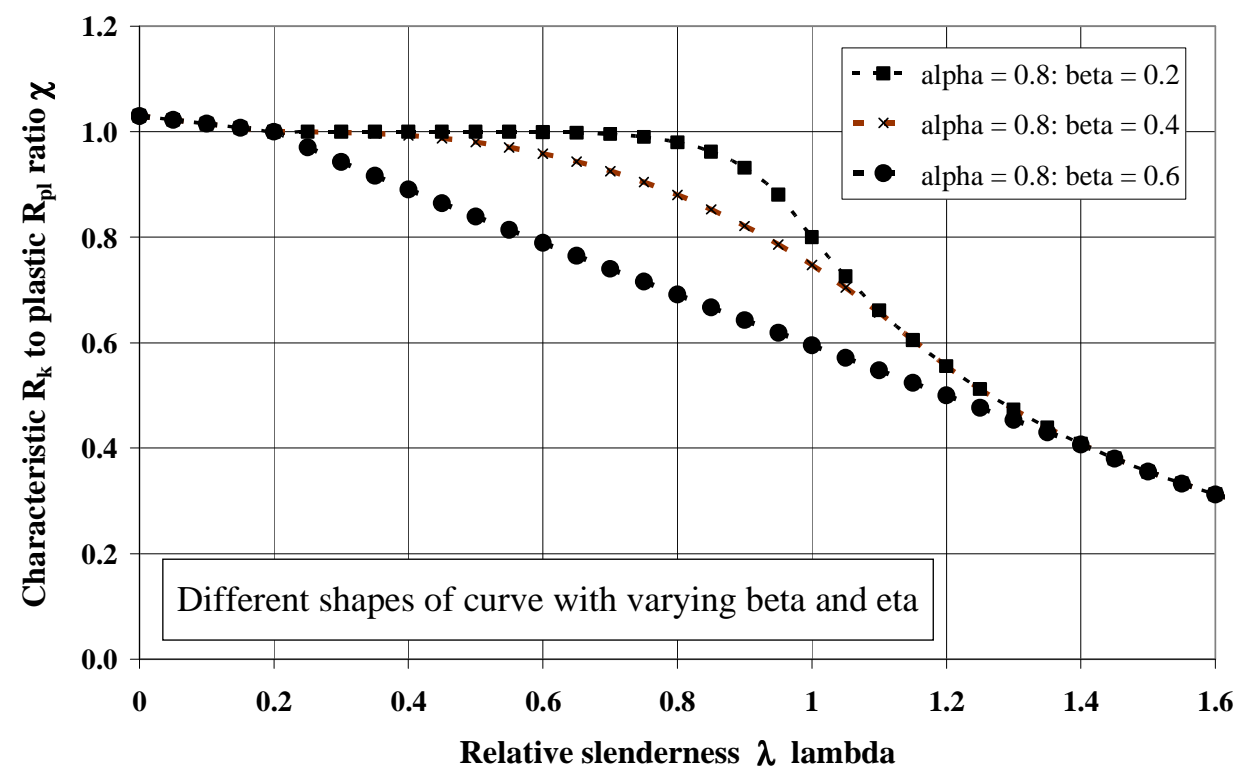

Figure 2: Curves with different sensitivity to plasticity [16]

\section{An improved portrayal of the capacity curve}

A serious disadvantage of the normal graphical representation of the capacity curve (Figures 1 and 2) is that the low resistances under elastic buckling conditions are very inaccurately represented, and the position of the change from elastic buckling to elasticplastic buckling is very difficult to determine accurately because a large percentage error in a small value is barely visible. This chiefly impinges on the assessed values of the elastic imperfection reduction factor $\alpha$ and the plastic range factor $\beta$. For structures that commonly lie in the plastic or relatively stocky range (columns and beams), this is not a serious drawback, but for structural systems that can be very slender (architectural novelties, spacecraft, vehicles, aircraft, silos and tanks), it presents a significant problem.

An easier extraction of these values [16] can be achieved when the $x$-axis is changed from 
relative slenderness $\lambda=\sqrt{R_{p l} / R_{c r}}$ to the relative generalized stiffness $R_{G M N I A} / R_{L B A}$ (Figure $3)$.

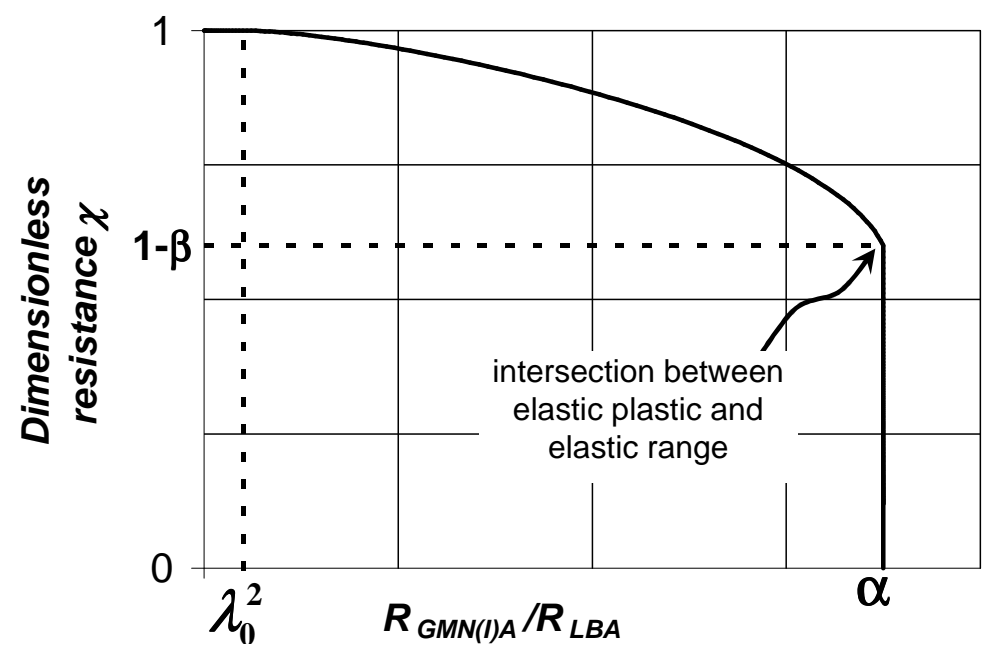

Figure 3: Modified capacity curve [16]

In the elastic range, the ratio $R_{G M N(I) A} / R_{L B A}$ is equal to the elastic imperfection reduction factor

$\frac{R_{G M N I A}}{R_{L B A}}=\alpha$

This means that this ratio $R_{G M N I A} / R_{L B A}$ is constant for a fixed value of $\alpha$, giving a vertical line (Figure 3), from which the elastic imperfection sensitivity factor $\alpha$ can be extracted easily. The position of the boundary between elastic buckling and elastic-plastic buckling is now also well defined (Figure 3) and the parameter $\beta$, which is governed by the load at which plasticity starts to affect the failure load significantly, can also be extracted easily. This arrangement of the traditional interaction curve, devised by [16], is particularly useful for the interpretation of computational predictions.

\section{Parametric studies involving a changing slenderness}

The full capacity curve, including both very slender and very stocky structures, can only be determined by changing the dimensionless slenderness $\lambda$ throughout a very wide range. 
Throughout most structural engineering studies in the past, the material properties were assumed to be predefined and the physical slenderness was changed by changing the geometry. For an axially compressed cylindrical shell this can be achieved by changing the radius to thickness ratio (Figure 4).

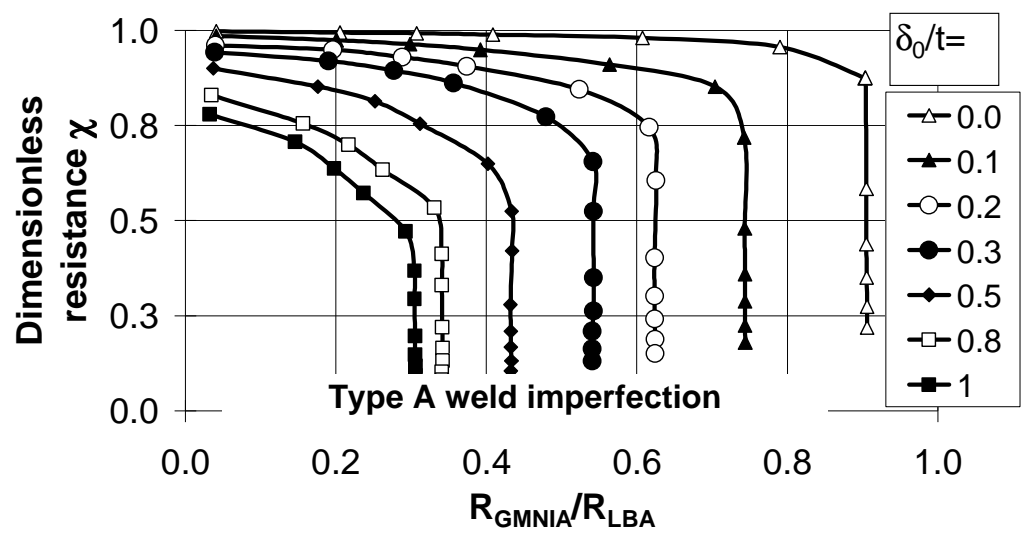

Figure 4: Modified capacity curves for imperfect cylinders under axial compression [17]

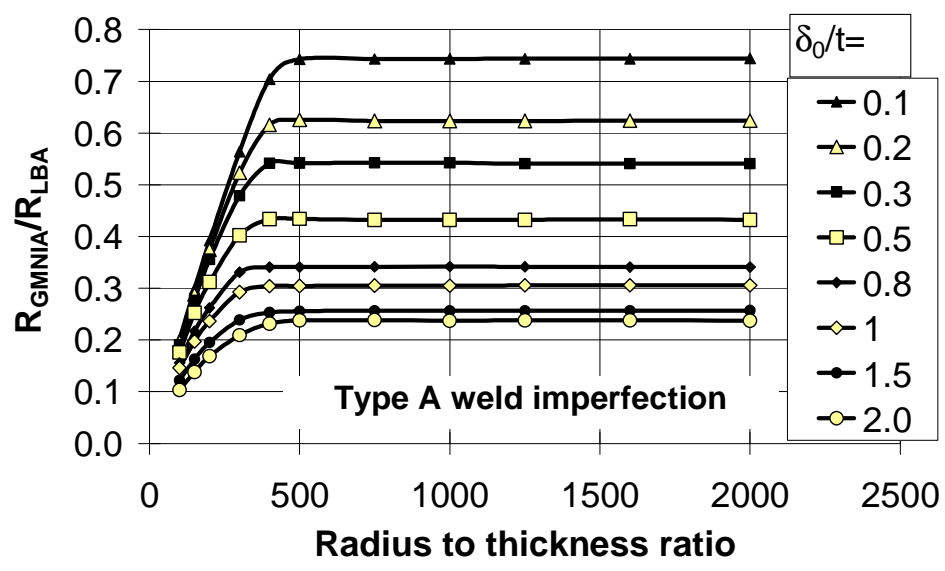

Figure 5: Axially compressed cylinder [18]

The elastic buckling ratio $R_{G N I A} / R_{L B A}$ (vertical line in the modified capacity curve) takes a value $\alpha$ that depends only on the imperfection form and amplitude for an axially compressed cylinder, and is independent of the geometric slenderness (Figure 5). Hence it is clear that the effect of geometric nonlinearity is independent of slenderness for a moderately long cylinder and the elastic imperfection factor $\alpha$ takes a constant value for a given imperfection amplitude. However, this is unfortunately a misleading example, because in most other situations the effect of geometric nonlinearity itself depends on the geometry (slenderness) of 
the structure, so $\alpha$ depends on both the geometric slenderness and the imperfection amplitude [19] and therefore it may not be possible to find a unique value of $\alpha$ (Figure 7).

That is to say in general, the value of $\alpha$ is likely to depend on the geometric parameters of the problem, but since it characterises elastic buckling, it cannot depend on the yield stress.

The chosen example geometry is a bracket supported perfect cylinder (Figure 6). Four brackets are attached to the cylinder at mid-height. The load is applied as tension on the bottom of the cylinder. For this example a bracket geometry of $h / \sqrt{r t}=5, d / \sqrt{r t}=1$. More detailed information on the modelling can be found in [20].

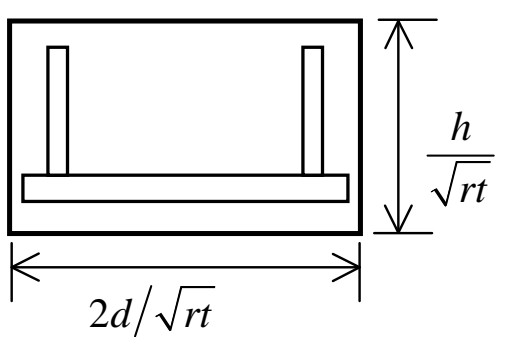

a) bracket dimensions

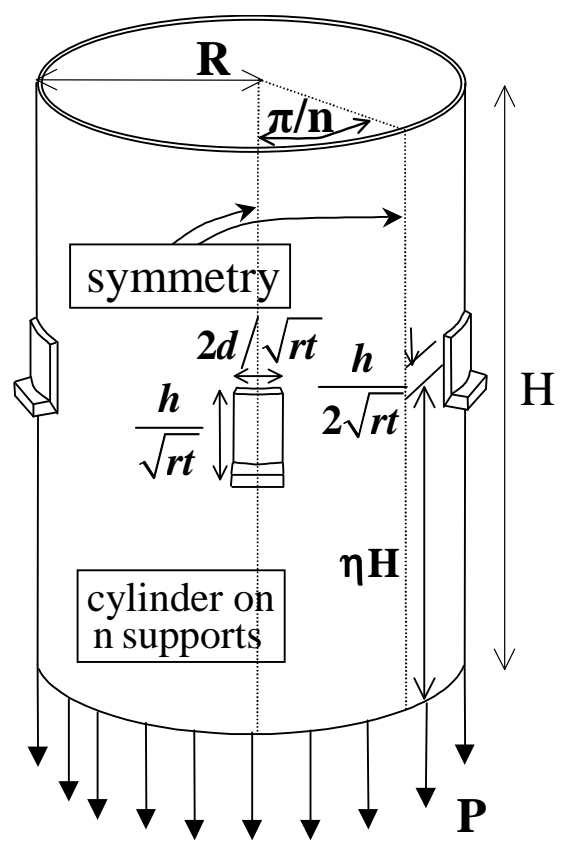

b) shell dimensions and notation

\section{Figure 6: Dimensions of a bracket supported silo}

The dependence of $\alpha$ on geometric parameters is illustrated in Figure 7, where the dimensionless slenderness of the bracket supported cylinder is changed by changing the radius to thickness ratio of the cylinder, but keeping values $h / \sqrt{r t}=5$ and $d / \sqrt{r t}=1$ throughout. It is apparent that the value of $\alpha$ is not constant as the radius to thickness ratio is changed. 


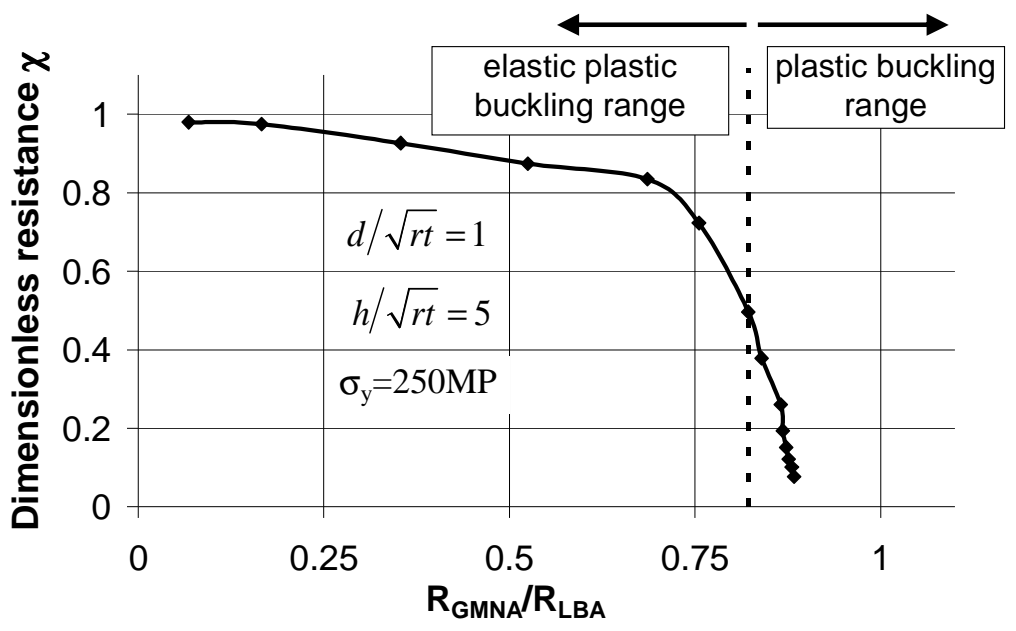

Figure 7: Modified capacity curve, changing r/t

In a more general case, one can expect that the value of $\alpha$ will depend on all geometric parameters and therefore a reliable calculation of the capacity curve can only be obtained by keeping the geometry constant in order to keep the geometric nonlinearity and imperfection sensitivity constant.

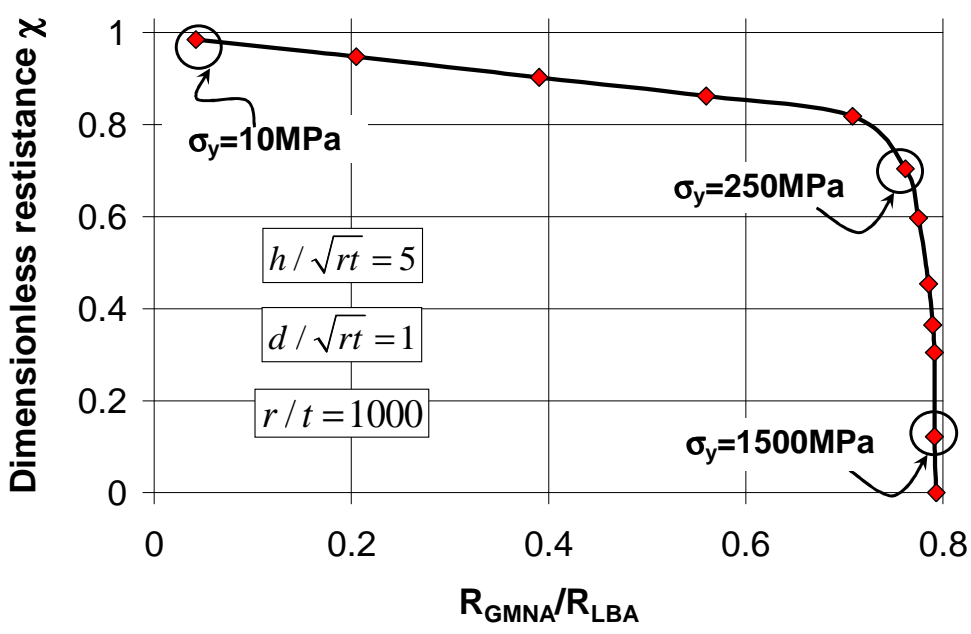

Figure 8: Modified capacity curve, changing $\sigma_{\mathrm{y}}$

The geometry can be kept constant and the dimensionless slenderness changed by changing the yield stress. This may sometimes involve a yield stress of unrealistic values (Figure 8), but it permits the complete mechanism of the problem to be found. Therefore this technique, first proposed by [21], was adopted in this investigation. 


\section{Capacity curves with constant $\eta$ and their restrictions}

In its original description [14], the parameter $\eta$ was assumed by default to take a constant value. For example, all interactions defined in EN 1993-1-6 [1] are set as $\eta=1$, based on limited experimental data and very simple lower bounds. This single value is derived from the German standard [22], which shows that it is over 20 years old and it does not include any of the research of the past 20 years.

However when the interaction exponent $\eta$ is assumed to remain constant, certain restrictions on the capacity curve apply. This impinges in particular on the intersection between the elastic plastic and elastic ranges of the capacity curve at $\lambda=\lambda_{p}$ (Figure 1 ).

The elastic section of the capacity curve defines the maximum buckling resistance without any degradation from the elastic material properties. It is therefore a necessary requirement that the elastic-plastic interaction defined by Eq. 4 must not produce resistances that exceed those of the elastic curve of Eq. 5.

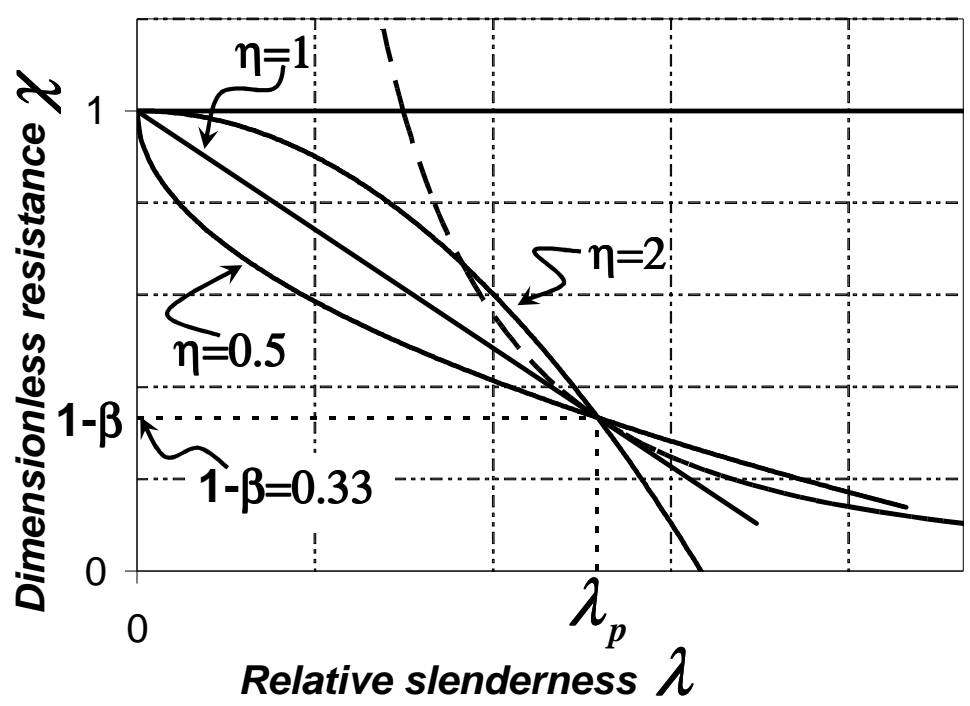




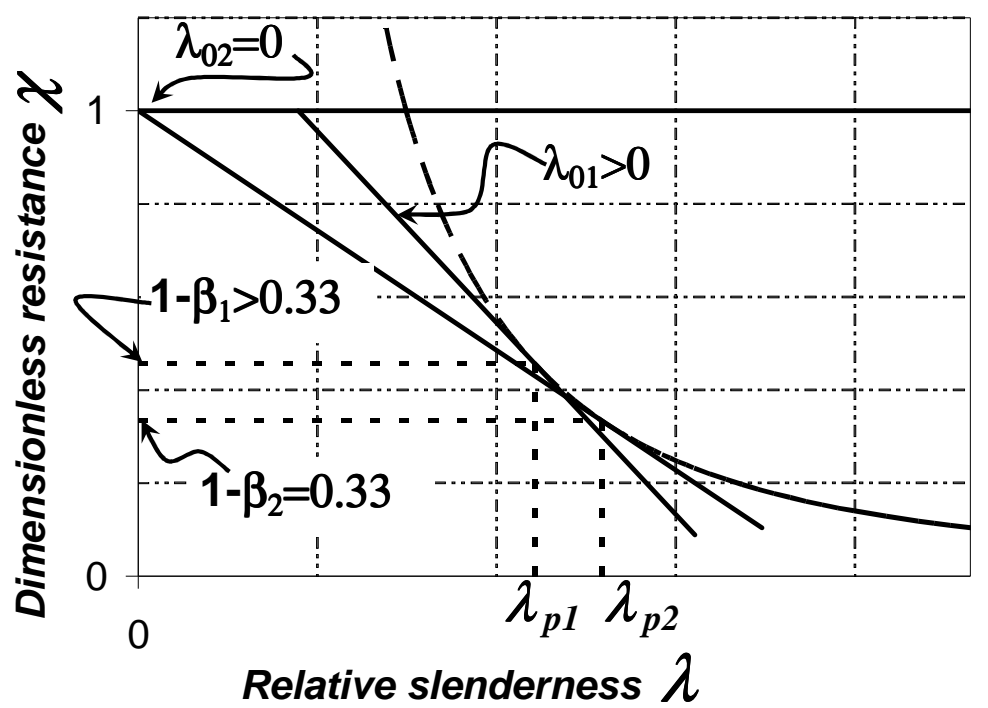

Figure 9: Value of $\beta$ is restricted by the requirement for tangency a) $\eta=$ constant, $\lambda_{0}=0$, b) $\eta=1, \lambda_{0}$ varying

When $\lambda_{0}=0$ and the parameter $\eta$ is larger than unity the resistance in the elastic plastic interaction range would be greater than the corresponding value for elastic material at the same slenderness. For $\eta<1$, the curvature of the elastic plastic interaction range changes and the capacity curve does not have the desired shape (

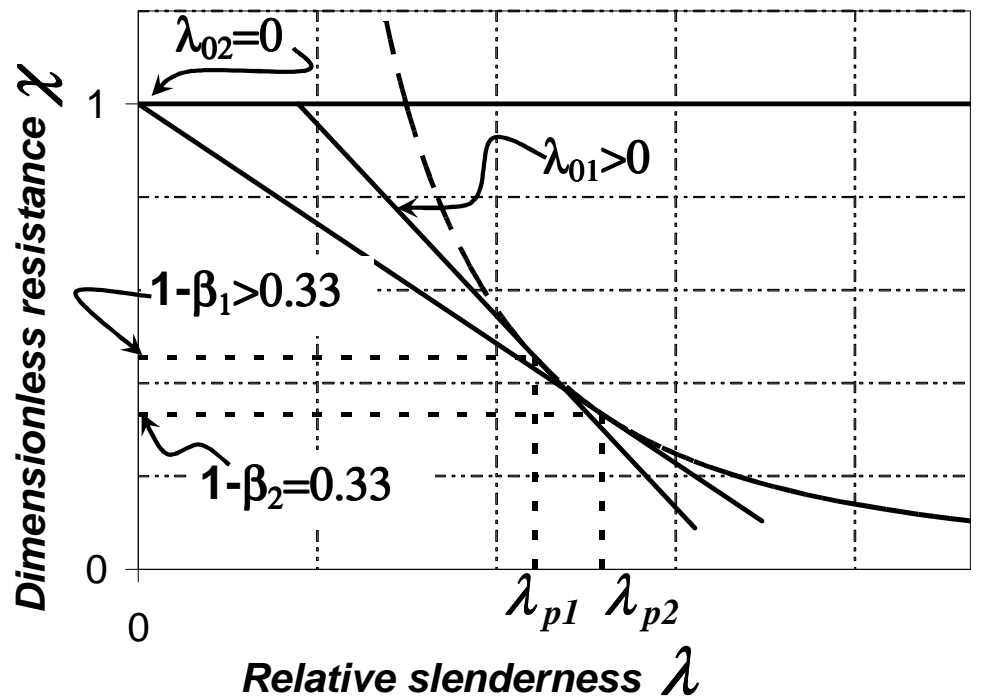

Figure 9a).

This requirement translates into two restrictions seen in the traditional capacity curve 
(Figure 1). First, the slope of the elastic-plastic interaction range at $\lambda=\lambda_{p}$ must be equal or smaller than the slope of the elastic range (Figure 10). Secondly the curvature of the elasticplastic interaction must not exceed that of the elastic value at this point (Figure 11). These two restrictions both limit the value of $\beta$ that can be adopted in Eqs 4 and 6, and thus restrict these expressions to structures in which yielding does not affect the buckling resistance until higher stress levels. The identification of these restrictions, and their elimination, are the goal of this paper.

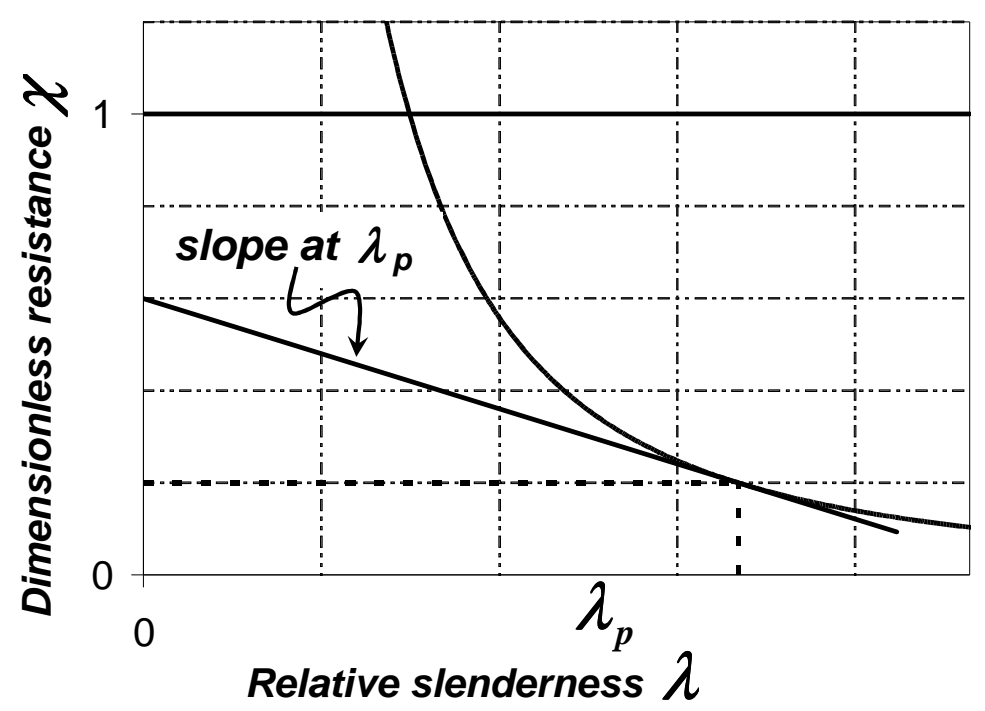

Figure 10: Restriction on the elastic-plastic slope at $\lambda=\lambda_{p}$

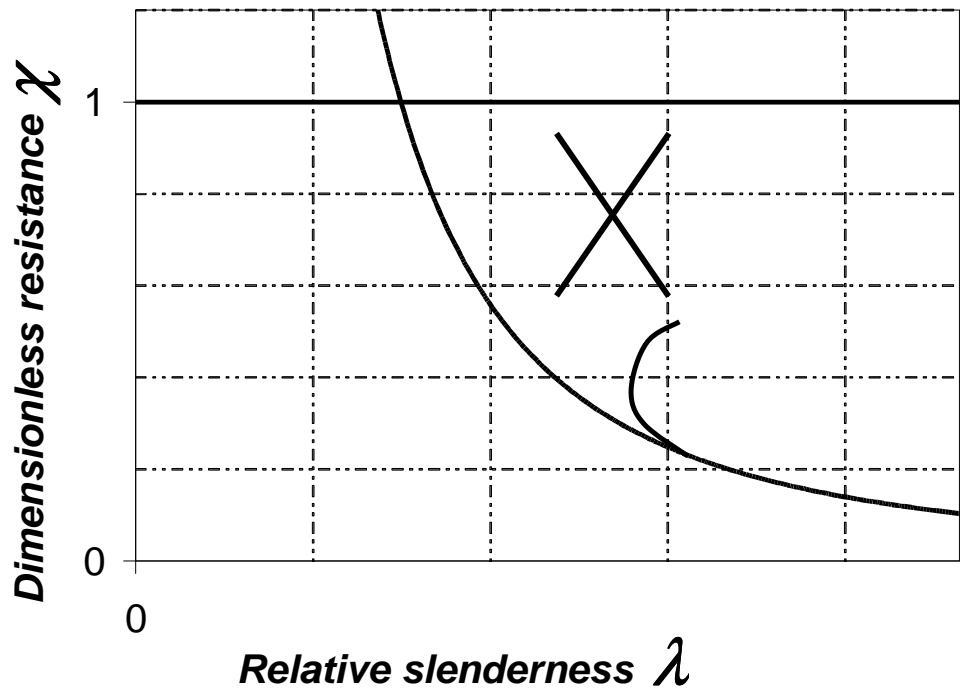

Figure 11: Restriction on the elastic-plastic curvature at $\lambda=\lambda_{p}$

Using the slope restriction identified above, the tangent to the elastic buckling curve at $\lambda_{p}$ 
must be more negative than the tangent to the elastic-plastic curve, or

$-\beta \frac{\eta}{\bar{\lambda}_{p}-\bar{\lambda}_{0}} \geq \frac{-2 \alpha}{\bar{\lambda}^{3}} \quad$ at $\lambda=\lambda_{\mathrm{p}}$

Or

$1-\beta \geq \frac{\eta \bar{\lambda}_{p}}{\left((\eta+2) \bar{\lambda}_{p}-2 \bar{\lambda}_{0}\right)}$

For a linear interaction coefficient $(\eta=1)$, the lowest possible value of $\chi$ at the onset of yielding (lowest $1-\beta$ ) occurs if there is no plastic plateau at all $\left(\bar{\lambda}_{0}=0\right)$ when the restriction becomes (

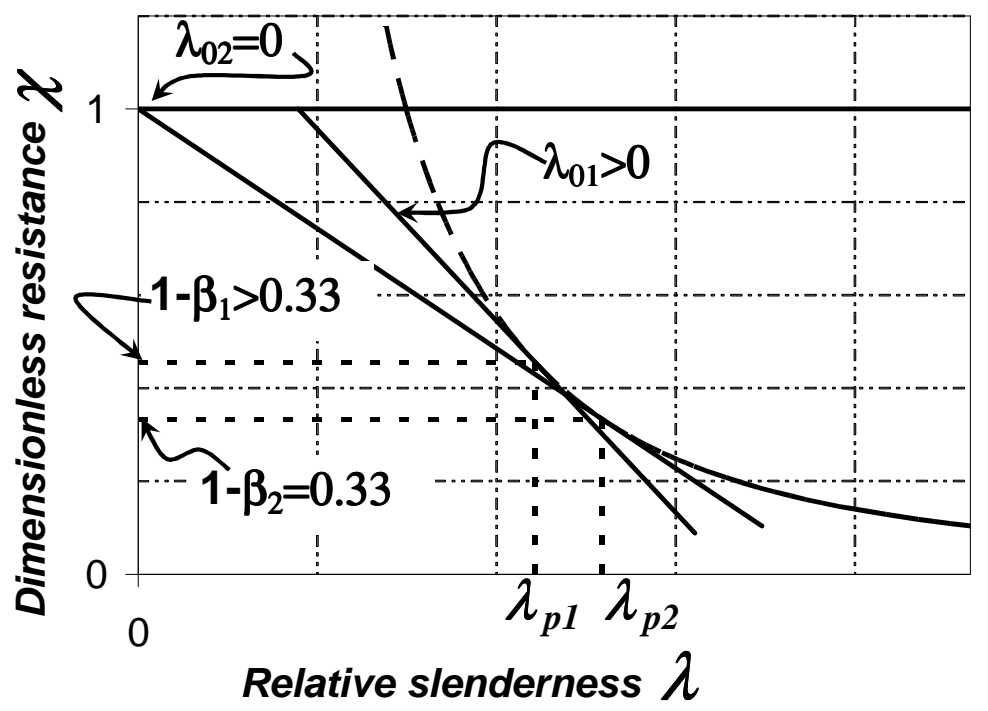

Figure 9b)

$1-\beta \geq 1 / 3$

Thus if $\eta=1$, no structures in which yielding affects the buckling resistance at loads lower that one third of the plastic collapse resistance can be well represented since yielding begins to affect the buckling resistance of many structures at a lower value than this. This restriction becomes more severe when a plastic plateau exists $\left(\bar{\lambda}_{0}>0\right)$ and, for a typical plateau $\left(\bar{\lambda}_{0}=0.2\right)$, this requirement restricts the onset of significant yielding to above $40 \%$ of the 
plastic collapse load. There are many structural arrangements in which yield affects the buckling resistance at low load levels, including tubes in bending and shell structures with high local stress raisers in the buckling zone.

\section{Capacity curves where $\eta$ varies linearly with $\lambda$}

To avoid this restriction on the plastic range factor $\beta$, it is proposed here that the interaction exponent $\eta$ should be assumed to vary linearly with the slenderness $\lambda$, so that Eq. 4 is altered into the form.

$\chi=1-\beta\left(\frac{\bar{\lambda}-\bar{\lambda}_{0}}{\bar{\lambda}_{p}-\bar{\lambda}_{0}}\right)^{\eta(\bar{\lambda})} \quad$ when $\quad \bar{\lambda}_{o}<\bar{\lambda}<\bar{\lambda}_{p}$

with

$\eta(\lambda)=\frac{\eta_{0}\left(\lambda_{p}-\lambda\right)+\eta_{p}\left(\lambda-\lambda_{0}\right)}{\lambda_{p}-\lambda_{0}}$

This expression delivers the interaction exponent $\eta=\eta_{0}$ at $\lambda_{0}$ and $\eta=\eta_{p}$ at $\lambda_{p}$, and permits the plastic limit interaction exponent $\eta_{p}$ to be smaller than unity where necessary. These limiting values of $\eta$ are hereafter termed the "plastic limit interaction exponent" $\eta_{p}$ found at the point where plastic behaviour begins, and the "squash limit interaction exponent" $\eta_{o}$, where the plastic collapse load is reached.

Equation 14 permits the onset of significant yielding, characterised by $1-\beta$ to take any value and does not restrict the length of the plastic plateau either. It also provides a much better fit to many interaction curves for structures in which local high stress concentrations arise [23]. However, the key aspects of slope and curvature limitations at $\lambda=\lambda_{p}$ must still be carefully observed, leading to the following limitations on the values that $\eta_{0}$ and $\eta_{p}$ may take so that $\beta$ may be chosen independently. 
When the parameters of the capacity are fitted to numerical or experimental results, it is expected that the elastic imperfection sensitivity factor $\alpha$ will be defined first, since it is only affected by the elastic behaviour and can be extracted easily from the modified capacity curve (Figure 3). The plastic range factor $\beta$ (onset of significant yielding) and squash limit relative slenderness $\lambda_{0}$ (end of the plastic plateau) can also be easily extracted from the numerical/experimental data.

The plastic limit relative slenderness $\lambda_{p}$ is determined directly from the parameters $\alpha$ and $\beta$ (Eq. 15).

$$
\lambda_{p}=\sqrt{\frac{\alpha}{1-\beta}}
$$

The only missing parameters now are the plastic limit interaction exponent $\eta_{p}$ and squash limit interaction exponent $\eta_{o}$. As noted above, both the slope and curvature of the elasticplastic range at $\lambda_{p}$ must be restricted to avoid higher predicted resistances in the elastic-plastic range than under elastic conditions.

To calculate the slope and curvature of the elastic plastic interaction range Equation 13 is rewritten as

$\chi(\lambda)=1-\beta u(\lambda)^{v(\lambda)}$

with

$$
\begin{aligned}
& u(\lambda)=\left(\frac{\lambda-\lambda_{0}}{\lambda_{p}-\lambda_{0}}\right) \\
& v(\lambda)=\frac{\eta_{p}\left(\lambda-\lambda_{0}\right)+\eta_{0}\left(\lambda_{p}-\lambda\right)}{\left(\lambda_{p}-\lambda_{0}\right)}
\end{aligned}
$$

The slope of the elastic plastic interaction range can then be expressed as 
$-\frac{1}{\beta} \chi^{\prime}(\lambda)=u(\lambda)^{v(\lambda)}\left[v^{\prime}(\lambda) \ln u(\lambda)+v(\lambda) \frac{u^{\prime}(\lambda)}{u(\lambda)}\right]$

and the curvature may be expressed as

$$
-\frac{1}{\beta} \chi^{\prime \prime}(\lambda)=u(\lambda)^{v(\lambda)}\left[\begin{array}{l}
\left(v^{\prime}(\lambda) \ln u(\lambda)+v(\lambda) \frac{u^{\prime}(\lambda)}{u(\lambda)}\right)^{2} \\
+v^{\prime \prime}(\lambda) \ln u(\lambda)+v^{\prime}(\lambda) \frac{u^{\prime}(\lambda)}{u(\lambda)} \\
+\frac{u(\lambda)\left\{v^{\prime}(\lambda) u^{\prime}(\lambda)+v(\lambda) u^{\prime \prime}(\lambda)\right\}-v(\lambda) u^{\prime}(\lambda)^{2}}{u(\lambda)^{2}}
\end{array}\right]
$$

At the critical point of interest, $\lambda=\lambda_{p}$, Equations $16-20$ take the values as follows

$$
\begin{aligned}
& u\left(\lambda_{p}\right)=\left(\frac{\lambda_{p}-\lambda_{0}}{\lambda_{p}-\lambda_{0}}\right)=1 \\
& u^{\prime}\left(\lambda_{p}\right)=\left(\frac{1}{\lambda_{p}-\lambda_{0}}\right) \\
& u^{\prime \prime}\left(\lambda_{p}\right)=0 \\
& v\left(\lambda_{p}\right)=\frac{\eta_{p}\left(\lambda_{p}-\lambda_{0}\right)+\eta_{0}\left(\lambda_{p}-\lambda_{p}\right)}{\left(\lambda_{p}-\lambda_{0}\right)}=\eta_{p} \\
& v^{\prime}\left(\lambda_{p}\right)=\frac{\eta_{p}-\eta_{0}}{\left(\lambda_{p}-\lambda_{0}\right)} \\
& v^{\prime \prime}\left(\lambda_{p}\right)=0
\end{aligned}
$$

Hence the slope of the elastic plastic curve at $\lambda=\lambda_{p}$ is given by

$$
\chi^{\prime}\left(\lambda_{p}\right)=-\beta\left(\frac{\eta_{p}}{\lambda_{p}-\lambda_{0}}\right)
$$

and the curvature at this point is given by 
$\chi^{\prime \prime}\left(\lambda_{p}\right)=\frac{-\beta}{\left(\lambda_{p}-\lambda_{0}\right)^{2}}\left(\eta_{p}^{2}+\eta_{p}-2 \eta_{0}\right)$

After the slope and the curvature of the elastic plastic curve are known, they can be compared with the slope and curvature of the elastic range. The slope of the elastic plastic range must be less negative than the slope of the elastic range. The limiting slope is shown in Figure 10, where the slope of the elastic plastic range at $\lambda_{p}$ is equal to the slope of the elastic curve.

The slope of the elastic buckling range be written as

$$
\chi^{\prime}(\lambda)=\frac{-2 \alpha}{\bar{\lambda}^{3}} \quad \text { when } \quad \bar{\lambda}_{p} \leq \bar{\lambda}
$$

and at $\lambda_{p}$ this becomes

$$
\chi^{\prime}\left(\lambda_{p}\right)=\frac{-2 \alpha}{\bar{\lambda}_{p}^{3}} \quad \text { when } \quad \bar{\lambda}_{p} \leq \bar{\lambda}
$$

Therefore the restriction for the limiting slope of the elastic plastic interaction range can be written as

$-\beta\left(\frac{\eta_{p}}{\lambda_{p}-\lambda_{0}}\right) \geq \frac{-2 \alpha}{\bar{\lambda}_{p}^{3}}=\frac{-2(1-\beta)}{\bar{\lambda}_{p}}$

which can be rearranged to give the restriction on $\eta_{p}$ in terms of $\beta$ and the two reference slendernesses

$$
\eta_{p} \leq 2\left(\frac{1-\beta}{\beta}\right)\left(\frac{\lambda_{p}-\lambda_{0}}{\lambda_{p}}\right)
$$

Since it is often better to have smooth transitions between one part of the capacity curve and another, it may be appropriate to insist that these two slopes (elastic and elastic-plastic) be equal at the point $\lambda=\lambda_{p}$. In this case, the inequality of Eq. 32 becomes an identity 
$\eta_{p}=2\left(\frac{1-\beta}{\beta}\right)\left(\frac{\lambda_{p}-\lambda_{0}}{\lambda_{p}}\right)$

which uniquely identifies the value of the plastic limit interaction exponent $\eta_{p}$ in terms of parameters which have already been determined.

The restriction on the curvature of the elastic-plastic zone leads to

$\frac{-\beta}{\left(\lambda_{p}-\lambda_{0}\right)^{2}}\left(\eta_{p}^{2}+\eta_{p}-2 \eta_{0}\right) \leq \frac{6 \alpha}{\bar{\lambda}_{p}^{4}}=\frac{6(1-\beta)}{\bar{\lambda}_{p}^{2}}$

which can be rearranged into the form

$\eta_{0} \leq 3\left[\frac{1-\beta}{\beta}\right]\left\{1-\frac{\lambda_{0}}{\lambda_{p}}\right\}^{2}+\frac{1}{2} \eta_{p}\left(1+\eta_{p}\right)$

Thus, the slope restriction only limits the value of $\eta_{p}$, whilst the curvature restriction also adds a limitation on the value of $\eta_{0}$. If $\eta_{\mathrm{p}}$ is determined to give slope continuity according to Eq. 33, then Eq. 35 simply limits the value of $\eta_{0}$.

These restrictions are generally not found to be particularly onerous, but this new formulation for the interaction exponent $\eta$ frees up the exploitation of the EN 1993-1-6 [1] stability expressions greatly. In particular, the freedom to permit $\eta$ to vary with $\lambda$ means that much more complicated shapes of elastic-plastic interactions can be accommodated, and much closer fits to detailed computational results can be achieved.

\section{Further possible desirable limitations}

It may be that it would be desirable to avoid two other forms of the elastic-plastic interaction curve. These are illustrated in Figure 12.

In the first, very early yielding (small value of $1-\beta$ ) may mean that it is desirable to demand that the slope be negative and the curvature positive at the point $\lambda=\lambda_{p}$. That is to say, 
the elastic-plastic line should not introduce falling resistances as the relative slenderness is reduced, and the sign of the curvature of this zone should match that of the elastic behaviour (Figure 12).

In the second, a very large value of $1-\beta$ arises when buckling and yielding do not interact very much at all, as for example in some cone-cylinder transition problems [24]. For this situation, it may be desirable to insist that the curvature of the elastic-plastic interaction is negative (Figure 12) at the point $\lambda=\lambda_{p}$.

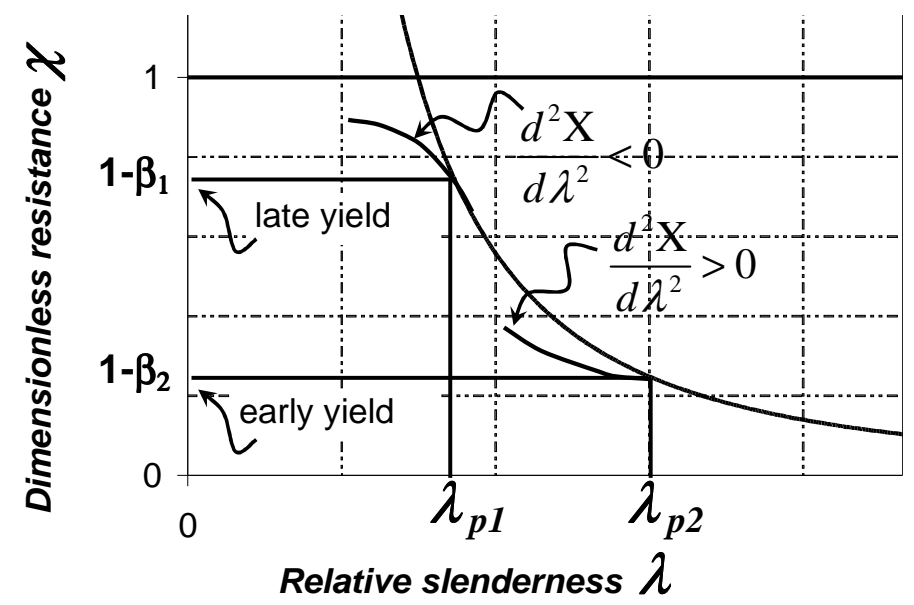

Figure 12: Possible useful additional restrictions on the form of the generalised capacity curve

In the first case, relating to early yielding situations, the slope of the elastic-plastic zone should be negative at $\lambda=\lambda_{p}$, leading to

$-\beta\left(\frac{\eta_{p}}{\lambda_{p}-\lambda_{0}}\right)<0$

which is always true provided $\eta_{p}>0$, so this is not a limitation at all.

The requirement that the curvature at this point should be positive leads to 
$\eta_{0}>\frac{1}{2}\left(\eta_{p}^{2}+\eta_{p}\right)$

For the second case, relating to very little elastic-plastic interaction, the requirement that the curvature of the elastic-plastic zone should be negative at $\lambda=\lambda_{p}$ leads naturally to the inverse condition

$\eta_{0}<\frac{1}{2}\left(\eta_{p}^{2}+\eta_{p}\right)$

These two restrictions are less critical than those given earlier and should only be applied with careful thought and used where the derived value of $\beta$ is appropriately high and low respectively.

\section{Application of the improved capacity curve}

As mentioned above, in the original proposals for this general capacity curve [25], the parameter $\eta$ was assumed by default to take a fixed value for given geometric and loading conditions. All interactions defined in EN 1993-1-6 [1] to date are set as $\eta=1$, based on limited experimental data and simple lower bounds. An interaction exponent of $\eta=1$ leads to a safe, but very uneconomical result (Figure 13) for a typical bracket supported cylinder (Figure $6)$. 


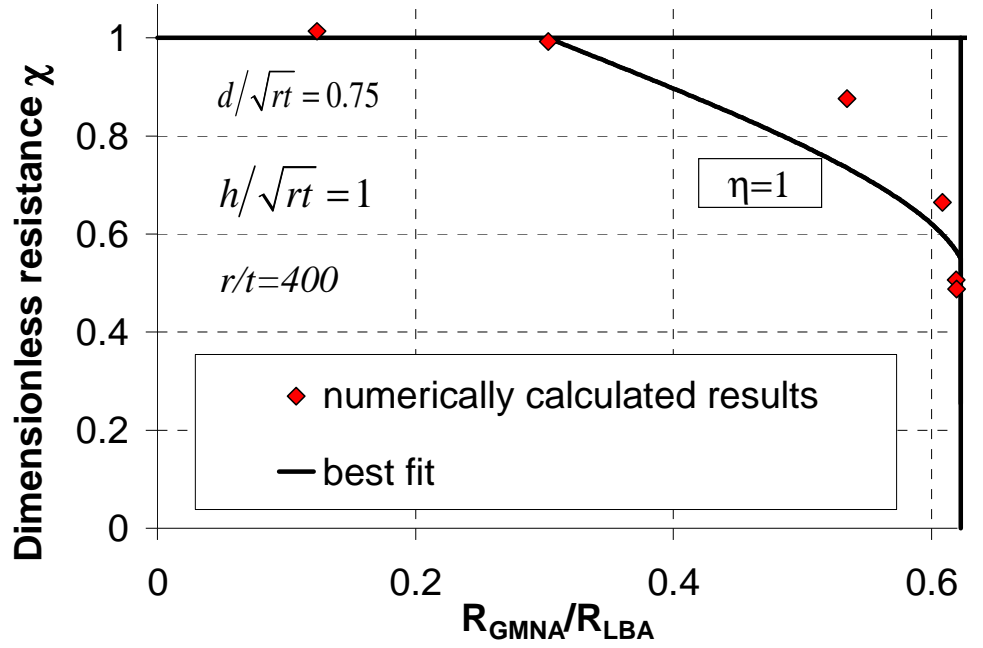

Figure 13: Modified capacity curve, $\eta=1.00$

An constant interaction exponent, which is not equal to one $(\eta \neq 1)$ results in an more economic result (Figure 14), but the linearly varying interaction exponent leads to an excellent results (Figure 15)

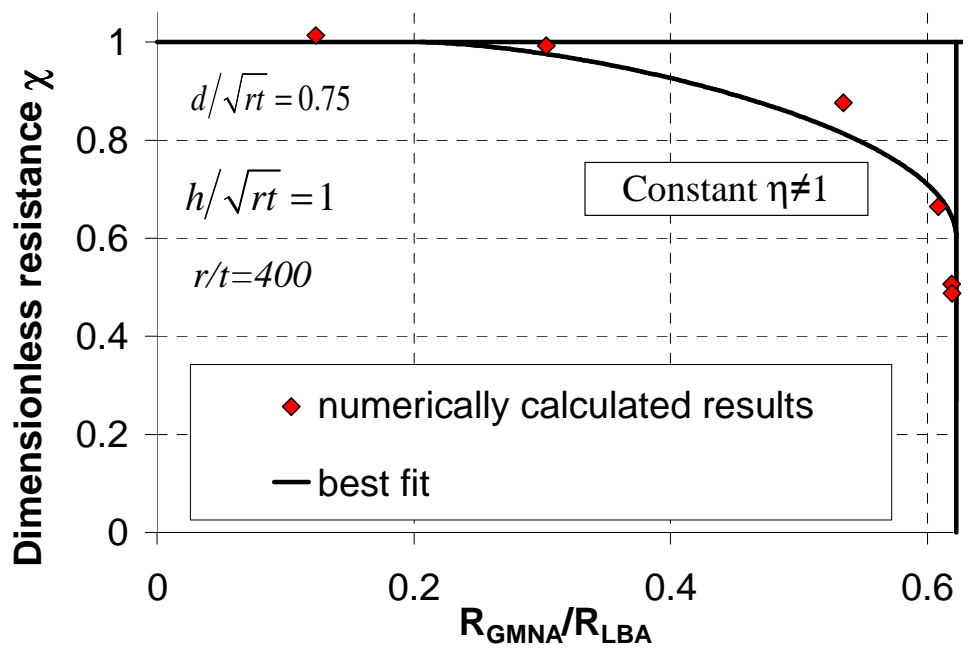

Figure 14: Modified capacity curve, $\eta=1.68$ 


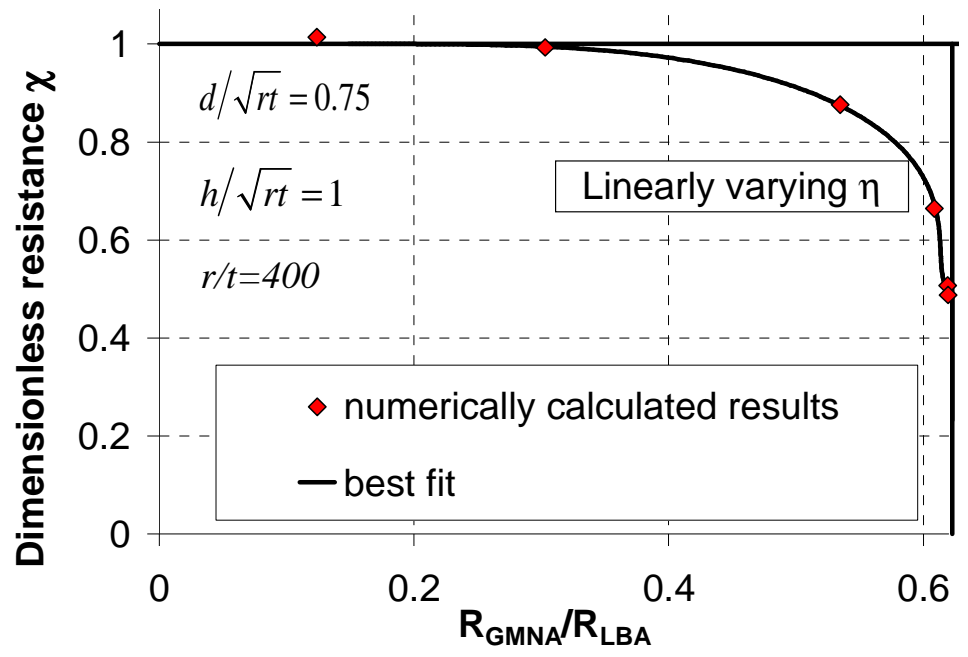

Figure 15: Modified capacity curve $\eta$ varies linearly with $\lambda$

The second example is a cylinder on engaged columns (Figure 16, $r / t=400, H / r=2$ ). Four columns are attached to the cylinder. The columns are engaged over a quarter of the cylinder height. The bottom and the top of the cylinder are restrained against out of round displacement and the columns are assumed to be rigid and fixed on the bottom. The rigidity of the columns is ensured by using rigid elements. The loading on the top of the cylinder is uniform axial compression. 


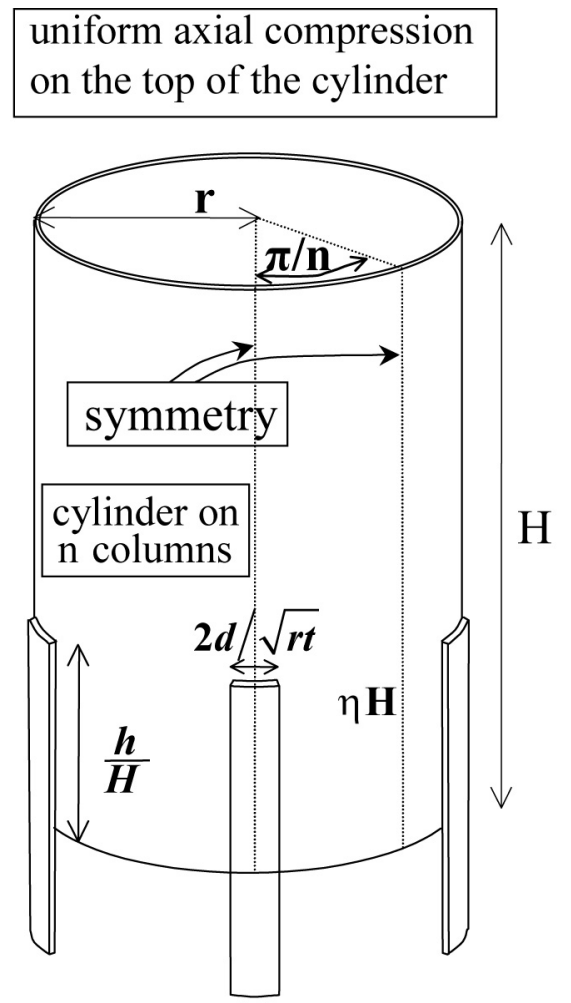

Figure 16: Cylinder on 4 engaged columns $(r / t=400, d / \sqrt{r t}=1.5, h / H=0.25, t=1)$

While the example of the bracket supported cylinder showed that the linearly varying interaction coefficient can be used to achieve a superior fit to numerical data than a merely constant interaction coefficient. The example of the cylinder on engaged column shows the true advantage of this new formulation. The numerical data yield a plastic range factor of $\beta=0.79$ or $1-\beta=0.21<1 / 3$. With a constant interaction coefficient of $\eta=1$ the restriction on the plastic range factor with $1-\beta>1 / 3$ still applies and hence the numerical data can not be fitted appropriately in the elastic plastic range close to the purely elastic range (Figure 17). Any larger constant interaction coefficient $\eta$ would yield an even more unsafe result with the above restrictions on the plastic range factor $\beta$. 


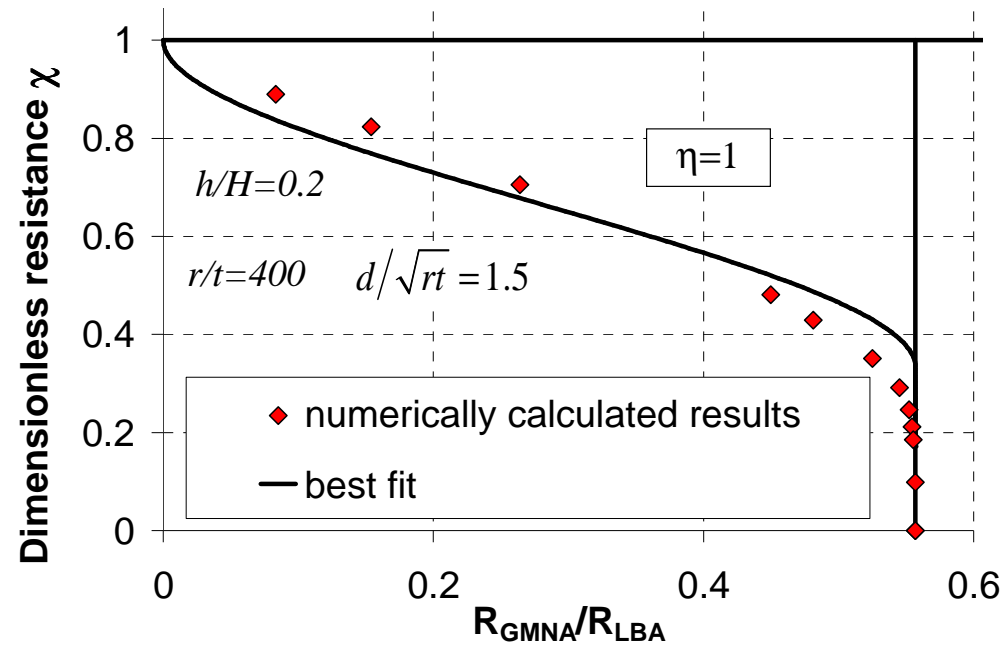

Figure 17: Modified capacity curve $\eta=1$ (engaged column)

However, using the proposed linearly varying interaction coefficient $\eta$, the data can be fitted appropriately without restriction on the plastic range factor $\beta$ (Figure 18).

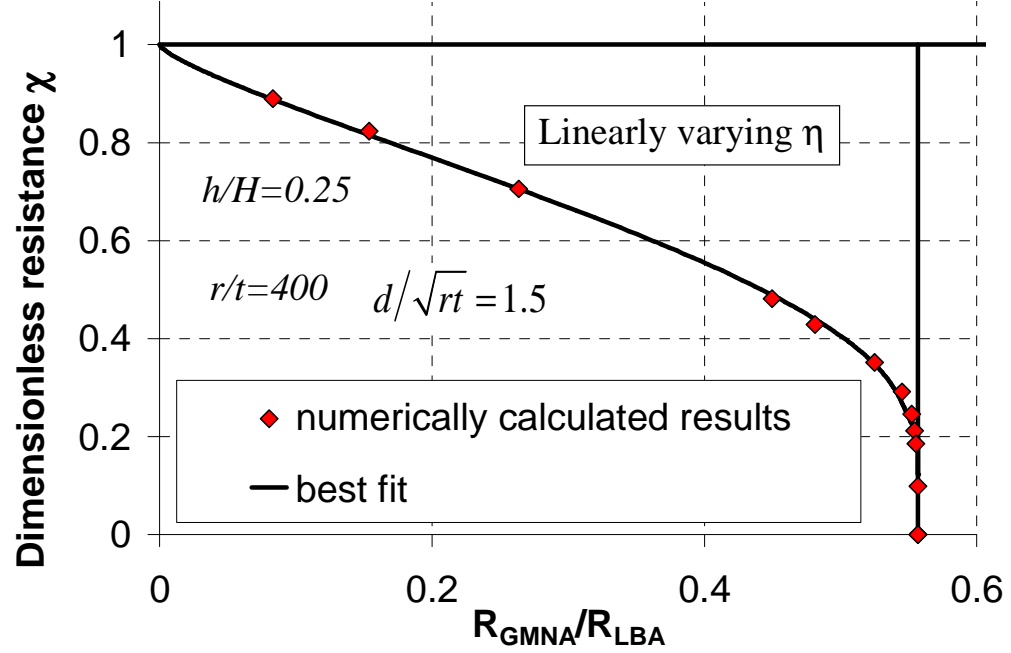

Figure 18: Modified capacity curve linearly varying $\eta$ (engaged column)

\section{Conclusions}

The current EN 1993-1-6 [1] capacity curve poses some unfortunate limitations on the values that key parameters may take. Although these restrictions are unimportant for many frame structures and shells under uniform stress fields, they often play a strong role when local stress concentrations cause early yielding. In this paper, these limitations are eliminated by making the interaction exponent $\eta$ vary with the relative slenderness $\lambda$. As a result, early yielding and better fits to numerical data for capacity curves can be achieved. However, the 
demands of appropriate shapes for the capacity curve impose some other restrictions on key values of $\eta$, and these have been identified and quantified in this paper.

\section{References}

[1] EN 1993-1-6, Eurocode 3: Design of steel structures: Part 1.6: General rules supplementary rules for the strength and stability of shell structures, Eurocode 3 Part 1.6, CEN, Brussels, 2007.

[2] J.M. Rotter, A framework for exploiting different computational assessments in structural design, 6th International Conderence on Steel and Aluminium Structures, 6th ICSAS 07, Oxford, 2007.

[3] J.M. Rotter, The Practical Design of Shell Structures Exploiting Different Methods of Analysis, SSTA8: Eighth Conference on Shell Structures: Theory and Applications, GdanskJurata, Taylor and Francis, London, 2005, pp. 71-86.

[4] EN 1993-1-1, Eurocode 3: Design of steel structures - Part 1-1: General Rules and Rules for Buildings, Eurocode 3 Part 1.1, CEN, Brussels, 2005.

[5] J.M. Rotter, H. Schmidt, Eds. Stability of Steel Shells: European Design Recommendations: Fifth Edition 2008, Publication P125, European Convention for Constructional Steelwork, Brussels, 2008.

[6] W.J.M. Rankine, Useful Rules and Tables, London, 1866.

[7] W.E. Ayrton, J. Perry, On struts, The Engineer, (1886), pp. 464-465, 513-515.

[8] A. Considère, Résistance des pièces comprimées, Congrès Internationale des Procédés de Construction, 1889, pp. 371.

[9] F. Engesser, Ueber die Knickfestigkeit gerader Staebe, Zeitschrift für Architekter und Ingenieurwsen, Verein zu Hannover, 35 (1889), pp. 455-562.

[10] A. Robertson, The Strength of Struts, Institution of Civil Engineers Selected Papers, London, 28 (1925), pp. 55.

[11] T.V. Galambos, R.L. Ketter, Columns under combined bending and thrust, Journal of the Engineering Mechanics Division, ASCE, Proc., 85 (1959), pp. 1-10.

[12] M. Beer, G. Schulz, Bases Theoretiques des Courbes Europeennes de Flambement, Construction Metallique, (1970), pp. 37-55.

[13] W. Merchant, The failure load of rigid jointed frameworks as influenced by stability, Structural Engineer, 32 (1954), pp. 185-190.

[14] J.M. Rotter, Proposal for generalisation of the elastic-plastic buckling interaction rule from Eurocode 3 Part 1.6, submission to CEN TC250/SC3/PT4 and ECCS TWG8.4 Buckling of Shells, (1999), pp. 8. 
[15] R. Greiner, P. Derler, Effect of imperfections on wind-loaded cylindrical shells, ThinWalled Structures, 23 (1995), pp. 271-281.

[16] J.M. Rotter, Shell Buckling and Collapse Analysis for Structural Design: The New Framework of the European Standard, in: H.R. Drew, S. Pellegrino (Eds.) New Approaches to Structural Mechanics, Shells and Biological Structures, Kluwer Academic Publishers, London, 2002, pp. 355-378.

[17] J.M. Rotter, The elastic-plastic imperfection sensitivity of axially compressed cylinders with weld depressions, Eurosteel 2008 Conference, Graz, Austria, 2008, pp. 1497-1502.

[18] J.M. Rotter, Design standards and calculations for imperfect pressurised axially compressed cylinders, Int. Conf. on Carrying Capacity of Steel Shell Structures, Brno, 1997, pp. 354-360.

[19] N. Yamaki, Elastic stability of circular cylindrical shells, North-Holland, 1984.

[20] C. Doerich, J.M. Rotter, Behavior of cylindrical steel shells supported on local brackets, Journal of Structural Engineering, 134 (2008), pp. 1269-1277.

[21] J.M. Rotter, Buckling Of Shallow Conical Shell Roofs For Small Diameter Tanks And Silos, International conference on design, inspection and maintenance of cylindrical steel tanks and pipelines, Prague, Czech Republic, 2003, pp. 169-175.

[22] DIN 18 800, Steel Structures: Stability, Buckling of Shells, DIN 18800 Part 4, Deutsches Institut für Normung, Berlin, 1990.

[23] C. Doerich, Strength and stability of locally supported cylinders, Institute for Infrastructure \& Environment, Edinburgh, University of Edinburgh, PhD, 2007: 350.

[24] J.G. Teng, Cone-cylinder intersection under internal pressure: nonsymmetric buckling, Journal of Engineering Mechanics, ASCE, 121 (1995), pp. 1298-1305.

[25] J.M. Rotter, Development of proposed European design rules for buckling of axially compressed cylinders, Advances in Structural Engineering, 1 (1998), pp. 273-286.

List of Figures:

Figure 1: Generalised capacity curve

Figure 2: Curves with different sensitivity to plasticity [16]

Figure 3: Modified capacity curve [16]

Figure 4: Modified capacity curves for imperfect cylinders under axial compression [17]

Figure 5: Axially compressed cylinder [18]

Figure 6: Dimensions of a bracket supported silo

Figure 7: Modified capacity curve, changing $r / t$ 
Figure 8: Modified capacity curve, changing $\sigma_{\mathrm{y}}$

Figure 9: Value of $\beta$ is restricted by the requirement for tangency a) $\eta=$ constant, $\lambda_{0}=0, b$ ) $\eta=1, \lambda_{0}$ varying

Figure 10: Restriction on the elastic-plastic slope at $\lambda=\lambda_{p}$

Figure 11: Restriction on the elastic-plastic curvature at $\lambda=\lambda_{p}$

Figure 12: Possible useful additional restrictions on the form of the generalised capacity curve

Figure 13: Modified capacity curve, $\eta=1.00$

Figure 14: Modified capacity curve, $\eta=1.68$

Figure 15: Modified capacity curve $\eta$ varies linearly with $\lambda$

Figure 16: Cylinder on 4 engaged columns $(r / t=400, d / \sqrt{r t}=1.5, h / H=0.25, \mathrm{t}=1)$

Figure 17: Modified capacity curve $\eta=1$ (engaged column)

Figure 18: Modified capacity curve linearly varying $\eta$ (engaged column) 